\title{
The Lowest Metallicity Stars from SDSS/SEGUE
}

\section{Timothy C. Beers}

JINA: Joint Institute for Nuclear Astrophysics, Dept. of Physics \& Astronomy, Michigan State University

E-mail: beers@pa.msu.edu

\section{Young Sun Lee}

JINA: Joint Institute for Nuclear Astrophysics, Dept. of Physics \& Astronomy, Michigan State University

E-mail: lee@pa.msu.edu

\section{Thirupathi Sivarani}

JINA: Joint Institute for Nuclear Astrophysics, Dept. of Physics \& Astronomy, Michigan State University, and Department of Astronomy, University of Florida

E-mail: thirupateastro.ufl.edu

\section{Daniela Carollo}

Research School of Astronomy and Astrophysics, Australian National University \& Mount Stromlo Observatory, and INAF-Osservatorio Astronomico di Torino, Italy

E-mail: carollo@mso.anu.edu.au

The first extension of the Sloan Digital Sky Survey (SDSS-II), which included the program SEGUE: Sloan Extension for Galactic Understanding and Exploration, ended in July, 2008. This effort, in which JINA was an institutional partner, has already produced the largest sample of medium-resolution stellar spectra yet obtained. These data are revolutionizing our understanding of the nature of the stellar populations of the Milky Way, in particular that of the halo populations.

The JINA-developed SEGUE Stellar Parameter Pipeline (SSPP) has obtained estimates of the atmospheric parameters $\left(\mathrm{T}_{\text {eff }}, \log \mathrm{g},[\mathrm{Fe} / \mathrm{H}]\right)$ for more than half of the spectra to date, totaling in excess of 200,000 stars. Here we report on the distribution of stellar metallicity, $[\mathrm{Fe} / \mathrm{H}]$, for stars in the SDSS/SEGUE database with $[\mathrm{Fe} / \mathrm{H}]<-2.0$. This sample now exceeds 16,000 stars, more than triple the number of such stars discovered by all previous surveys combined. We also comment on the prospects for further increases in this sample in the near future, from the next extension of SDSS (SDSS-III), which will include a full year of SEGUE operation from July 2008 to July 2009, and additional "piggy back" observations of stars taken during subsequent years.

10th Symposium on Nuclei in the Cosmos

July 27 - August 1, 2008

Mackinac Island, Michigan, USA

\footnotetext{
${ }^{*}$ Speaker.
} 


\section{Introduction}

We are now firmly entrenched in the era of massive photometric and spectroscopic surveys of the stellar populations of the Galaxy, as highlighted by the Sloan Digital Sky Survey (SDSS-I; [1]), and its first extension, SDSS-II, as well as by the contemporaneous survey RAVE [2]. These surveys, and others that are planned for the near future, promise to completely revolutionize our understanding of the formation and evolution of the Milky Way, and provide new insight into the formation of the chemical elements. Here we concentrate on the low-metallicity stars found during the course of SDSS/SEGUE, the shape of their metallicity distribution below $[\mathrm{Fe} / \mathrm{H}]=-2.0$, and on the implications of the recently confirmed inner/outer halo structure of the Galaxy for future searches for these stars.

\section{SEGUE}

The SEGUE survey took its last data in June, 2008. Over 3600 square degrees of new imaging was obtained, and a total of over 400 spectroscopic plug-plates were observed (each plug-plate has 600 science fibers and some 40 sky/calibration fibers), covering some 200 lines of sight probing directions of interest for exploration of the thick disk and halo populations of the Milky Way, from $0.5 \mathrm{kpc}$ to almost $100 \mathrm{kpc}$ from the Sun (see Figure 1). The medium-resolution $(R=2000)$ spectroscopy obtained for roughly 240,000 program stars (in the magnitude range $14.0<g<20.5$ ), in combination with the ugriz photometry from the imaging, form the inputs for determinations of radial velocities (accurate to between 5 and $25 \mathrm{~km} / \mathrm{s}$, depending on the color of the star and the S/N of the spectrum) and, most crucially, the fundamental stellar atmospheric parameters $\left(\mathrm{T}_{\mathrm{eff}}, \log \mathrm{g}\right.$, and $[\mathrm{Fe} / \mathrm{H}]$ ) for the majority of the program objects. The full data set (and derived parameters for the stars) from SDSS-I and SDSS-II is planned to be released to the public in fall 2008.

The atmospheric parameters are derived from a set of techniques, based on a number of different calibrations, in an effort to provide robust determinations that remain valid over the large range of parameter space and S/N explored by SEGUE. These approaches, which collectively are applied by the SEGUE Stellar Parameter Pipeline (SSPP), include techniques for finding the minimum distance (parameterized in various ways) between observed spectra and grids of synthetic spectra (e.g., [4]), non-linear regression models (e.g., [5], and references therein), correlations between broadband colors and the strength of prominent metallic lines, such as the CaII K line [6], auto-correlation analysis of a stellar spectrum ([6], and references therein), obtaining fits of spectral lines (or summed line indices) as a function of broadband colors [7]), or the behavior of the CaII triplet lines as a function of broadband color [8].

Each of the methods employed by the SSPP exhibit optimal behavior over restricted temperature and metallicity ranges; outside of these regions they are often un-calibrated, suffer from saturation of the metallic lines used in their estimates at high metallicity or low temperatures, or lose efficacy due to the weakening of metallic species at low metallicity or high temperatures. The techniques that make use of specific spectral features are susceptible to other problems, e.g., the presence of emission in the core of the CaII K line for chromospherically active stars, or poor telluric line subtraction in the region of the CaII triplet. Because SDSS stellar spectra cover most of the entire optical wavelength range, one can apply several approaches, using different wavelength 


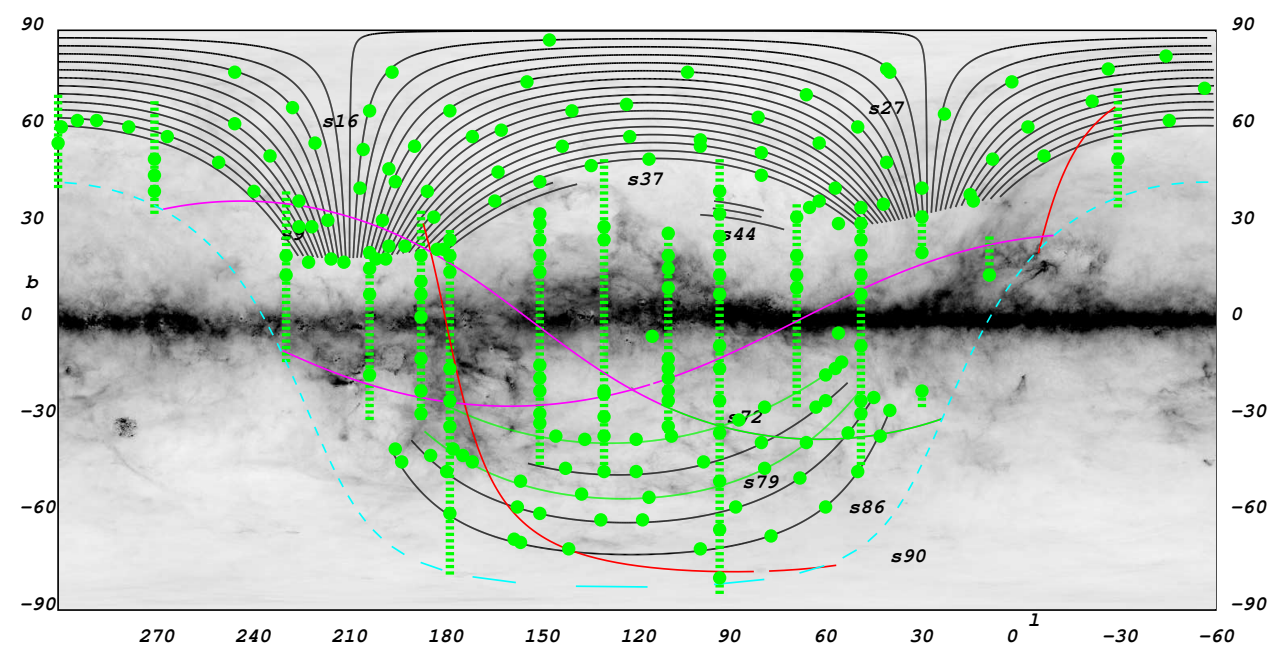

Figure 1: Map of the imaging and spectroscopic coverage obtained during the SDSS/SEGUE program. The black lines correspond to regions imaged during SDSS-I, while the colored lines indicate regions of SEGUE imaging. The green filled dots indicate lines of sight where SEGUE obtained over 1200 individual spectra (in two spectroscopic plug-plates, a bright plate and a faint plate). The image of the Milky Way is derived from the dust maps of [3].

regions, in order to glean optimal information on stellar parameters. The combination of multiple techniques results in estimates of stellar parameters that are more robust over a much wider range of $\mathrm{T}_{\text {eff }}, \log \mathrm{g}$, and $[\mathrm{Fe} / \mathrm{H}]$ than those that might be produced by individual methods.

Details of these procedures and tests of the validity of the resulting parameter estimates are presented in a series of three papers $([9,10,11])$, to which the interested reader is referred.

The precision of the parameter estimates varies with the $\mathrm{S} / \mathrm{N}$ of the spectra. At the median $\mathrm{S} / \mathrm{N}$ of the SEGUE spectra (roughly 20/1), the estimates have typical errors of $\delta \mathrm{T}_{\text {eff }}=150 \mathrm{~K}, \delta \log \mathrm{g}=$ $0.30 \mathrm{dex}$, and $\delta[\mathrm{Fe} / \mathrm{H}]=0.25 \mathrm{dex}$, respectively. Tests on the accuracy of the atmospheric parameter estimates indicate that there exist negligible zero-point offsets over the majority of the parameter space.

\section{Why the Fascination with Large Numbers of Metal-Poor Stars?}

In the grand timeline of the formation of structure in the Universe, the very first luminous objects to have formed are thought to be massive stars. These objects, the so-called First Stars, are expected to have had masses in the range from several tens to hundreds of times that of the Sun. After brief lifetimes (no more than a few million years) these stars exploded, likely seeding the intergalactic medium with the very first nuclei of elements heavier than $\mathrm{H}$ and $\mathrm{He}$. Although studies of the First Stars and First Galaxies themselves remain the territory of theoreticians, and many fine reviews are available that discuss their formation and evolution (see, e.g., $[12,13])$, there now exists a growing database of observational information that constrains many of their properties.

The First Stars are expected to have left their "chemical fingerprints" on the early generations of lower-mass stars that formed from the (slightly) enriched gas that they left behind at their demise. 
These low-mass stellar populations are likely to have included objects of sufficient long mainsequence lifetimes that they can be identified to the present day, some 13 billion years after their formation. It is these stars that form the basis for our present discussion. Once the very first low-mass stars form, they also serve as "tracer particles" of their parent structures. In the $\Lambda$ CDM paradigm, these stars were likely to have been born in the proto-Galactic clumps of dark matter and gas that eventually coalesced to form the full-fledged galaxies we see today, including the Milky Way. Their present spatial distributions and kinematical properties retain information on their birthplaces; when combined with detailed chemical abundance measurements, their study can reveal much about the manner and timescales over which galaxies form and grow.

It is thus expected that the shape of the low-metallicity tail of the Metallicity Distribution Function (MDF) will (eventually) show structure that reveals the characteristic metallicities associated with major epochs of star formation in the Galaxy. Furthermore, the change in the MDF as a function of distance will constrain the assembly history of the Milky Way, and other large spirals like it. In addition, determinations of the frequency of various elemental abundance signatures, such as enhancements of $[\mathrm{C} / \mathrm{Fe}]$, and measures of $[\alpha / \mathrm{Fe}]$ ratios, will provide information on the nature of the star formation histories of the proto-Galactic fragments, most of which have already been incorporated into the Milky Way, and on their accretion timescales. It is also of great interest to nuclear astrophysics to identify the relatively rare objects found among samples of very metalpoor stars, such as stars with clear evidence of enhancements of the elements associated with the $\mathrm{s}$ (low)- and $\mathrm{r}$ (apid) neutron-capture processes which account for the production of essentially all of the elements beyond the iron peak. The small handful of stars known today with measurable uranium and thorium, and which can be used to infer direct ages of their progenitor objects through cosmo-chronometry, are also of keen interest (see Frebel, this volume).

As the database of low-metallicity stars continues to grow, it becomes increasingly important to develop and refine numerical models of Galactic Chemical Evolution [14]. The real power of these approaches will come from a closer coupling with the expected elemental yields of the First Stars, as well as with the expected kinematics that results from the capture of proto-Galactic fragments in the still-assembling Galaxy.

\section{Previous Searches for Metal-Poor Stars and the SDSS/SEGUE Effort}

Metal-poor stars in the Galaxy are quite rare, compared to those with metallicities approaching solar. It has been estimated that the fraction of stars within a few $\mathrm{kpc}$ of the Sun with metallicities below $[\mathrm{Fe} / \mathrm{H}]=-2.0$ (the Very Metal-Poor [VMP] stars according to the nomenclature of [15]) represent less than $0.1 \%$ of a volume-limited sample. Those with lower metallicities are rarer still. Hence the first challenge that must be faced is to efficiently identify these "diamonds of the night sky."

The effect that metallic lines have on the emergent flux from a star can be exploited to search for stars of low metallicity [16]. Although the de-blanketing effect (as measured with broad-band filters) nearly vanishes below $[\mathrm{Fe} / \mathrm{H}]=-2.0$, one may still make use of the technique to separate stars that are likely to be above or below this value, at least within the color range where it applies.

The SDSS has already generated the largest homogeneous catalog of well-calibrated broadband photometry for Galactic stars, using the broad-band ugriz system [17]. Such data now exist 
Table 1: Comparison of Metal-Poor Stars Discovered Pre- and Post-SDSS

\begin{tabular}{lccr}
\hline Category & Abbreviation & Metallicity & Number (Pre/Post) \\
\hline Metal-Poor & MP & {$[\mathrm{Fe} / \mathrm{H}]<-1.0$} & $10,000+/ 100,000+$ \\
Very Metal-Poor & VMP & {$[\mathrm{Fe} / \mathrm{H}]<-2.0$} & $4,000+/ 20,000+$ \\
Extrememly Metal-Poor & EMP & {$[\mathrm{Fe} / \mathrm{H}]<-3.0$} & $200+/ 400+$ \\
Ultra Metal-Poor & UMP & {$[\mathrm{Fe} / \mathrm{H}]<-4.0$} & $5 / 5$ \\
Hyper Metal-Poor & HMP & {$[\mathrm{Fe} / \mathrm{H}]<-5.0$} & $2 / 2$ \\
Mega Metal-Poor & MMP & {$[\mathrm{Fe} / \mathrm{H}]<-6.0$} & $0 / 0$ \\
\hline
\end{tabular}

for over 200 million stars [1]. The utility of the SDSS photometric system for the identification of low-metallicity stars has been explored already by [18] and [19]. Due to the use of broad-band filters, and the typical errors in the photometry obtained during the SDSS survey (a few percent), the SDSS system is less than ideal for this application. However, large numbers of candidates can be potentially generated. Hence, if highly multiplexed spectrographs are employed for obtaining follow-up verification (as is done for a subset of SDSS stars during the SEGUE program), the efficiency of candidate selection is not a critical limitation.

In Table 1, we contrast the total numbers of metal-poor stars (with varying degrees of metal deficiency) discovered during the course of all previous searches conducted over the past half century with the numbers of metal-poor stars identified during the course of SDSS/SEGUE. Over 90\% of the stars contained in the previous samples come from two sources, the HK survey of Beers and colleagues [20] and the Hamburg/ESO survey (HES) of Christlieb and colleagures [21]. As can be seen, the numbers of metal-poor stars identified by SDSS/SEGUE dominates the samples from previous surveys for the MP and VMP categories, is roughly similar to the previous numbers of stars known with metallicities at the EMP level. It is also clear that, at least so far, SDSS/SEGUE has not added any (confirmed) stars in the UMP and HMP categories. This is not unexpected, since high-resolution follow-up of the lowest metallicity stars discovered during SDSS/SEGUE has only commenced in the past year or so.

Because there are certain to exist biases in total SDSS/SEGUE sample, due to the color-based selection against stars with abundances above $[\mathrm{Fe} / \mathrm{H}]=-2.0$ in some of the categories, Figure 2 shows the MDF of F-,G-, and K-type stars from this survey with $[\mathrm{Fe} / \mathrm{H}]<-2.0$.

\section{The Inner/Outer Halo Structure of the Galaxy}

Although it had been long speculated that the halo of the Milky Way might be structurally complex, and comprise more than a single stellar population, the calibration stars from SDSS/SEGUE have been used to demonstrate convincingly that the halo is indeed clearly divisible into two broadly overlapping structural components, an inner and an outer halo, which exhibit different spatial density profiles, stellar orbits, and stellar metallicities [22]. While the inner halo has a modest net prograde rotation (now estimated to be $20 \pm 5 \mathrm{~km} / \mathrm{s}$ ), the outer halo exhibits a net retrograde rotation (now estimated to be $-85 \pm 9 \mathrm{~km} / \mathrm{s}$ ), and a peak metallicity three times lower $([\mathrm{Fe} / \mathrm{H}]=$ $-2.2)$ than that of the inner halo $([\mathrm{Fe} / \mathrm{H}]=-1.6)$. These properties indicate that the individual halo 
components likely formed in fundamentally different ways, possibly through successive dissipational (inner) and dissipationless (outer) mergers and the tidal disruption of proto-Galactic clumps. Work is now in progress to derive the velocity ellipsoids and metallicity distribution functions of the individual populations.

The application of these decomposition models to the DR-6 calibration stars can also be used to derive the approximate fractions of stars contributed to the Galaxy from each stellar population. While the thick disk and inner halo dominate within $5 \mathrm{kpc}$ of the plane, the inner halo dominates between 5 and $15 \mathrm{kpc}$ from the plane, and the outer halo dominates beyond $15 \mathrm{kpc}$.

The implications for the existence of the inner/outer halo dichotomy are likely to be profound. For example, the dominance of the inner-halo population in the solar neighborhood may account for the difficulty in the identification of very metal-poor stars with magnitude limited objectiveprism surveys, such as the HK survey and the HES, as well for the fact that the most extreme such stars found to date have all come from the HES, which explores up to two magnitudes fainter (and is less biased against cooler, more distant giants) than the HK survey.

The clear difference in the MDFs of the two halo populations also suggests that the lowest metallicity stars in the Galaxy may well be associated with the outer halo, which can be exploited in future directed searches. It is noteworthy that the hyper metal-poor stars HE 0107-5240 [23] and HE 1327-2326 [24], both of which have $[\mathrm{Fe} / \mathrm{H}]<-5.0$, as well as the recently discovered ultra metal-poor star HE 0557-4840 [25], with $[\mathrm{Fe} / \mathrm{H}]=-4.8$, are either located greater than 10 kpc away (HE 0107-5240, HE 0557-4840) or have space motions that carry them far out into the Galaxy (HE 1327-2326). This information could be used to search for the most metal-poor stars in future surveys, by selection of stars with highly retrograde proper motions, or with sufficiently high proper motions to indicate that they are on highly energetic orbits.

Efforts to determine the primordial lithium abundance from observations of the most metal-

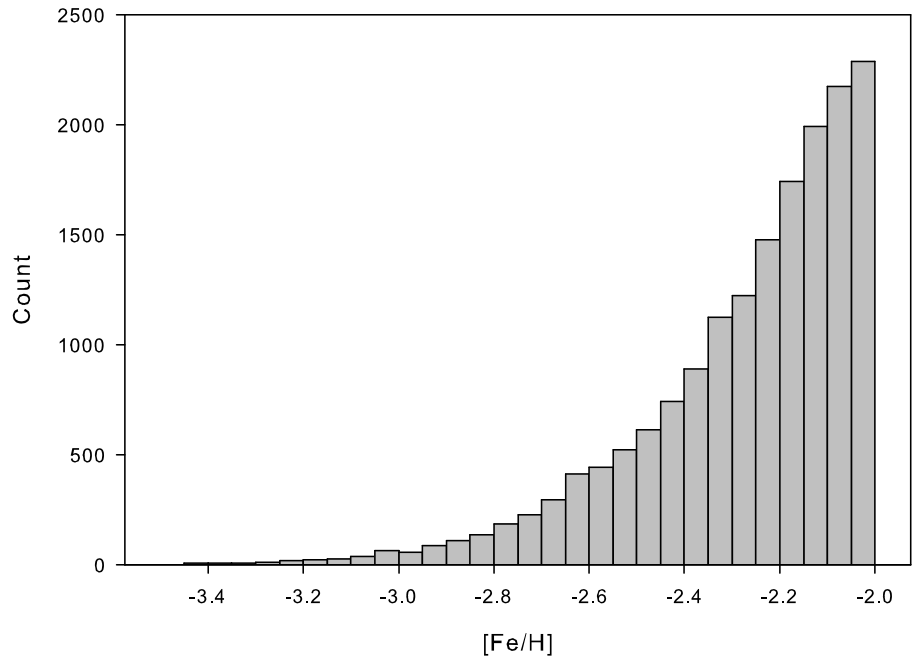

Figure 2: The Metallicity Distribution Function of over 16000 F-G-K stars from SDSS/SEGUE with [Fe/H] $<-2.0$. 
poor stars (e.g., [26]) may have inadvertently mixed samples from the inner- and outer-halo populations; such stars could have formed and evolved in rather different astrophysical environments. The inner/outer halo dichotomy may also have an impact of the expected numbers of carbon-enhanced metal-poor stars as a function of declining metallicity [27], and as a function of distance from the Galactic plane [28, 29].

\section{A Metallicity Map of the Disks and Inner Halo}

One recent application of the SDSS photometry has been the construction of a metallicity map for millions of stars in the disk and halo populations of the Milky Way [30]. Based on the spectroscopically calibrated stars from SDSS/SEGUE, these authors have derived photometric estimates of $[\mathrm{Fe} / \mathrm{H}]$ for some 2.5 million F-type stars in the color range $0.2<g-r<0.4$, with accuracies only slightly less than the spectroscopic determinations, and exploring distances up to $9 \mathrm{kpc}$ from the Sun. Such a technique will be used in the future, e.g., with data from LSST and SkyMapper, to obtain metallicity maps of the halo of the Galaxy out to $100,000 \mathrm{kpc}$, with substantially improved accuracy. See Figure 3.

\section{Future Surveys}

SEGUE-2 is one of four surveys planned as part of the next extension to the SDSS, known as SDSS-III, which began in July, 2008 and will run through 2014. SEGUE-2 will use the existing SDSS spectrograph to obtain medium-resolution spectra for roughly an additional 200,000 stars, during a period of one year starting in August, 2008. Building on what has been learned from SEGUE-1, SEGUE-2 will specifically target stars (in a variety of categories) that explore the transition from the inner to the outer halo, concentrating on stars at the main-sequence turnoff, distant giants and horizontal-branch stars, and on stars selected to be likely low-metallicity objects.

There exist a number of large high-resolution spectroscopic surveys that are just now getting underway, which should greatly enlarge the numbers of VMP stars with available elemental abundance information. The Chemical Abundances of Halo Stars (CASH) survey is making use of the Hobby-Eberly Telescope to obtain moderately high-resolution $(R=15000)$ spectroscopy for up to 1,000 VMP stars identified during the course of the HK-I, HK-II, and HES efforts, with a large number of additional stars from SDSS/SEGUE. Aoki and collaborators have recently been awarded Key Project status on the Subaru Telescope in order to obtain $R=50000$ spectroscopic observations for up to 200 VMP stars, the majority of which will be drawn from SDSS/SEGUE targets. Both of these surveys have as one of their primary aims to test for the existence (or not) of chemical signatures that might be associated with the inner/outer halo dichotomy [22].

The Apache Point Observatory Galactic Evolution Experiment (APOGEE), another survey to be conducted as part of SDSS-III, will use high-resolution $(R=20000)$, high signal-to-noise ratio ( $\mathrm{S} / \mathrm{N}=100 / 1), \mathrm{H}$-band $(1.6 \mu \mathrm{m})$ spectroscopy to penetrate the dust that obscures the inner Galaxy from our view, observing 100,000 red giant stars across the full range of the Galactic bulge, bar, disk, and halos. The high spectral resolution of APOGEE will allow element-by-element measure-

ments of chemical abundances, which can be used to reconstruct the history of star formation that 


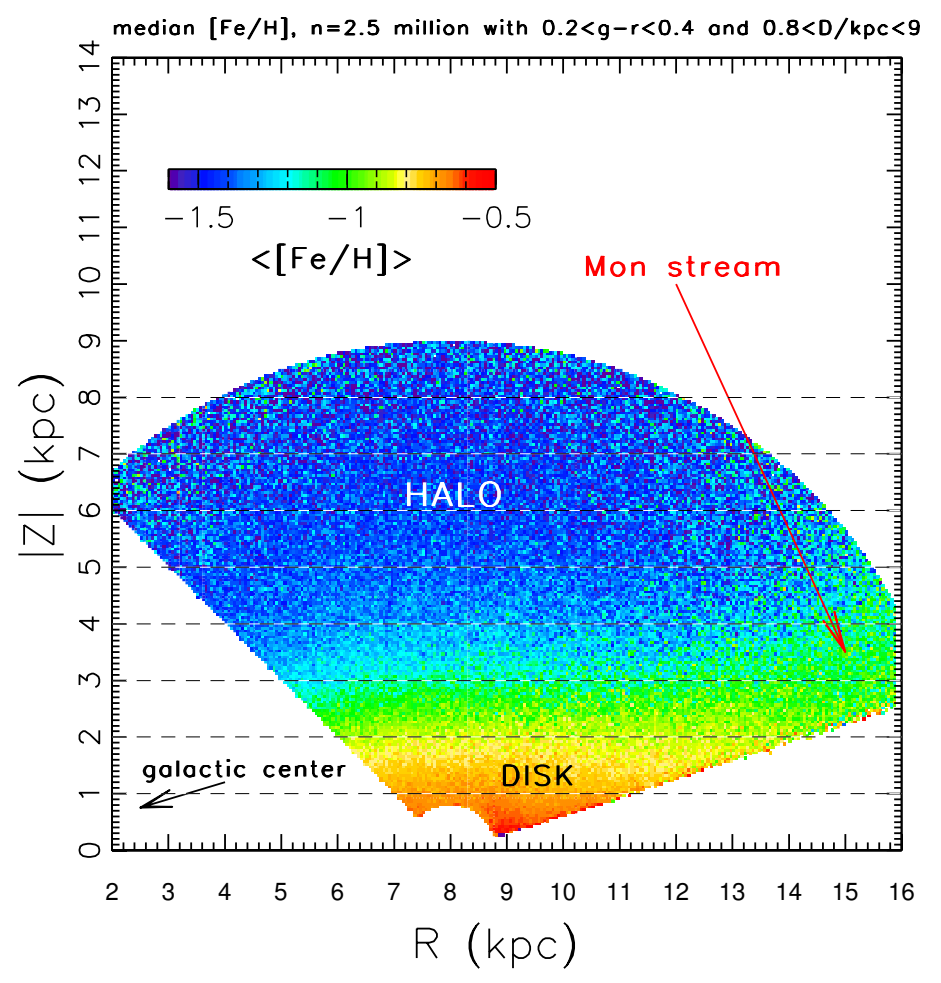

Figure 3: Photometric metallicities for 2.5 million blue F-type stars, based on the calibration described by [30]. The median occupancy of the 40,000 pixels shown in this map is 33 stars, with a minimum of 5 stars per pixel. The color-coded metallicities clearly indicate the presence of the disk, thick-disk, and inner-halo populations of the Galaxy. The presence of the Monoceros stream in the region close to the disk plane is indicated.

produced these elements. Together, SEGUE-2 and APOGEE will provide a picture of the Milky Way that is unprecedented in scope, richness, and detail.

The Large Area Multi-Object Spectroscopy Telescope (LAMOST) project, funded by the Chinese National Academy of Science, will employ a massive 4000 fiber system mounted on a widefield $4 \mathrm{~m}$ meridian Schmidt telescope. It is expected that this ambitious project will obtain up to several million medium-resolution $(R=2000)$ spectroscopic observations of stars in the Milky Way and Local Group galaxies. With the planned addition of $R=5000 / 10000$ gratings, follow-up observations at moderately high spectral resolution will also become possible.

Of course, we all look forward to the possible execution of the WFMOS (Wide Field MultiObject Spectrograph) survey of up to one million stars at resolving power $R=50000$. Currently, this Gemini instrument is expected to be mounted on the prime focus of the Subaru telescope, in 
order to take advantage of its wide field of view. The hope, and expectation, is that this survey will finally reveal the rich set of elemental abundances for stars that probe the entire history of chemical evolution throughout the Galaxy.

\section{Acknowledgments}

Funding for the SDSS and SDSS-II has been provided by the Alfred P. Sloan Foundation, the Participating Institutions, the National Science Foundation, the U.S. Department of Energy, the National Aeronautics and Space Administration, the Japanese Monbukagakusho, the Max Planck Society, and the Higher Education Funding Council for England. The SDSS Web Site is http://www.sdss.org/.

This work received partial support from grants AST 07-07776 and PHY 02-15783; Physics Frontier Center / Joint Institute for Nuclear Astrophysics (JINA), awarded by the US National Science Foundation.

\section{References}

[1] Adelman-McCarthy, J.K, Agüeros, M.A., Allah, S.S, Allende Prieto, C., Anderson, K.S.J., et al. 2008, The Sixth Data Release of the Sloan Digital Sky Survey, ApJS, 175, 297

[2] Zwitter, T., Siebert, A., Munari, U., Freeman, K.C.. Siviero, A., Watson, F. G., et al. 2008, The Radial Velocity Experiment (RAVE): Second Data Release, AJ, 136, 421

[3] Schlegel, D.J., Finkbeiner, D.P., \& Davis, M. 1998, Maps of Dust Infrared Emission for Use in Estimation of Reddening and Cosmic Microwave Background Radiation Foregrounds, ApJ, 500, 525

[4] Allende Prieto, C., Beers, T.C., Wilhelm, R., Newberg, H.J., Rockosi, C.M., et al. 2006, A Spectroscopic Study of the Ancient Milky Way: F- and G-Type Stars in the Third Data Release of the Sloan Digital Sky Survey, ApJ, 636, 804

[5] Re Fiorentin, P., Bailer-Jones, C.A.L., Lee, Y.S., Beers, T.C., Sivarani, T., et al. 2007, Estimation of Stellar Atmospheric Parameters from SDSS/SEGUE Spectra, A\&A, 467, 1373

[6] Beers, T.C., Rossi, S., Norris, J.E., Ryan, S.G., \& Shefler, T. 1999, Estimation of Stellar Metal Abundance. II. A Recalibration of the Ca II K Technique, and the Autocorrelation Function Method $A J, 117,981$

[7] Wilhelm, R., Beers, T.C., \& Gray, R.O. 1999, Spectroscopy of Hot Stars in the Galactic Halo. II. The Identification and Classification of Horizontal-Branch and Other A-Type Stars, AJ, 117, 2308

[8] Cenarro, A.J., Cardiel, N, Gorgas, J., Peletier, R.F., Vazdekis, A., \& Prada, F. 2001, Empirical Calibration of the Near-infrared Ca II triplet - I. The Stellar Library and Index Definition, MNRAS, 326, 959

[9] Lee, Y.S., Beers, T.C., Sivarani, T., Allende Prieto, C., Koesterke, L., et al. 2008, The SEGUE Stellar Parameter Pipeline. I. Description and Initial Validation Tests, AJ, in press (arXiv:0710.5645)

[10] Lee, Y.S., Beers, T.C., Sivarani, T., Johnson, J.A., An, D., et al. 2008, The SEGUE Stellar Parameter Pipeline. II. Validation with Galactic Globular and Open Clusters, AJ, in press (arXiv:0710.5778)

[11] Allende Prieto, C., Sivarani, T., Beers, T.C., Lee, Y.S., Koesterke, L. et al. 2008, The SEGUE Stellar Parameter Pipeline. III. Comparison with High-Resolution Spectroscopy of SDSS/SEGUE Field Stars, $A J$, in press (arXiv:0710.5780) 
[12] O’Shea, B.W., McKee, C.F., Heger, A., \& Abel, T. 2008, Conference Summary, in First Stars III, eds. B.W. O’Shea, A. Heger, \& T. Abel, AIP Conference Proceedings 990, p. xiii (arXiv:0801.2124)

[13] Grief, T.H., Johnson, J.L., Klessen, R.S., \& Bromm, V. 2008, The First Galaxies: Assembly, Cooling, and the Onset of Turbulence, MNRAS, 387, 1021

[14] Tumlinson, J. 2006, Chemical Evolution in Hierarchical Models of Cosmic Structure. I. Constraints on the Early Stellar Initial Mass Function, ApJ, 641, 1

[15] Beers, T.C. \& Christlieb, N. The Discovery and Analysis of Very Metal-Poor Stars in the Galaxy, $A R A A, \mathbf{4 3}, 531$

[16] Sandage, A. 1969, New Subdwarfs. II. Radial Velocities, Photometry, and Preliminary Space Motions for 112 Stars with Large Proper Motion, ApJ, 158, 1115

[17] Ivezic, Z., Lupton, R.H., Schlegel, D., Boroski, B., Adelman-McCarthy, J., Yanny, B., et al. 2004, SDSS Data Management and Photometric Quality Assessment, AN, 325, 583

[18] Lenz, D.D., Newberg, H.J, Rosner, R., Richards, G.T., \& Stoughton, C. 1998, Photometric Separation of Stellar Properties Using SDSS Filters, ApJS, 119, 121

[19] Helmi, A., Ivezic, Z., Prada, F., Pentericci, L., Rockosi, C.M., Schneider, D.P., et al. 2003, Selection of Metal-Poor Giant Stars Using the Sloan Digital Sky Survey Photometric System, ApJ, 586, 195

[20] Beers, T.C., Preston, G.W., and Shectman, S.A., A Search for Stars of Very Low Metal Abundance. II., $A J, \mathbf{1 0 3}, 1987$

[21] Christlieb, N., Finding the Most Metal-poor Stars of the Galactic Halo with the Hamburg/ESO Objective-prism Survey, RvMA, 16, 191

[22] Carollo, D., Beers, T.C., Lee, Y.S., Chiba, M., Norris, J.E., et al. 2007, Two Stellar Components in the Halo of the Milky Way, Nature, 450, 1020

[23] Christlieb, N., Bessell, M.S., Beers, T.C., Gustafsson, B., Korn, A., et al. 2002, A Stellar Relic From the Early Milky Way, Nature, 419, 904

[24] Frebel, A., Aoki, W., Christlieb, N., Ando, H., Asplund, M., Barklem, P.S., et al. 2005, Nucleosynthetic Signatures of the First Stars, Nature, 434, 871

[25] Norris, J.E., Christlieb, N., Korn, A.J., Eriksson, K., Bessell, M. S., Beers, T.C., et al. 2007, HE 0557-4840: Ultra-Metal-Poor and Carbon-Rich, ApJ, 670, 774

[26] Bonifacio, P., Molaro, P., Sivarani, T., Cayrel, R., Spite, M., Spite, F., et al. 2007, First Stars VII Lithium in Extremely Metal-Poor Dwarfs, A\&A, 462, 851

[27] Lucatello, S., Beers, T.C., Christlieb, N., Barklem, P.S., Rossi, S., Marsteller, B., et al. 2006, The Frequency of Carbon-enhanced Metal-poor Stars in the Galaxy from the HERES Sample, ApJ, 653, L37

[28] Frebel, A., Christlieb, N., Norris, J.E., Beers, T.C., Bessell, M.S., Rhee, J., et al. 2006, Bright Metal-Poor Stars from the Hamburg/ESO Survey. I. Selection and Follow-up Observations from 329 Fields, ApJ, 652, 1585

[29] Tumlinson, J. 2007, Carbon-Enhanced Metal-Poor Stars, the Cosmic Microwave Background, and the Stellar Initial Mass Function in the Early Universe, ApJ, 664, L63

[30] Ivezic, Z., Sesar, B., Juric, M., Bond, N., Dalcanton, J., Rockosi, C.M., et al. 2008, The Milky Way Tomography with SDSS: II. Stellar Metallicity, ApJ, 684, 287 